\title{
shRNA targeting PLK1 inhibits the proliferation and invasion of nasopharyngeal carcinoma cells
}

\author{
Yan Zhou ${ }^{1,2}$, Chu Wu ${ }^{1}$, Bingxue Liu ${ }^{1}$, Juan Zhu ${ }^{1}$, Yating Zhong ${ }^{3}$, Yuqing Yuan ${ }^{1}$, Yue Huang ${ }^{1}$, Yunlian Tang ${ }^{1}$ \\ ${ }^{1}$ Cancer Research Institute, Medical College of Hengyang, Key Laboratory of Tumor Cellular \& Molecular Pathology, University of South China, \\ Hengyang, China; ${ }^{2}$ Department of Pathology, People's Hospital of Ningxiang, Changsha, China; ${ }^{3}$ Department of Pathology, The First People's \\ Hospital of Changde City, Changde, China \\ Contributions: (I) Conception and design: Y Tang, Y Zhong, Y Zhou; (II) Administrative support: Y Tang; (III) Provision of study materials or \\ patients: Y Zhou, C Wu, J Zhu, Y Huang; (IV) Collection and assembly of data: C Wu, B Liu, Y Yuan; (V) Data analysis and interpretation: C Wu, B \\ Liu, Y Yuan; (VI) Manuscript writing: All authors; (VII) Final approval of manuscript: All authors. \\ Correspondence to: Dr. Yunlian Tang. Cancer Research Institute, Medical College of Hengyang, Key Laboratory of Tumor Cellular \& Molecular \\ Pathology, University of South China, Hengyang 421001, China. Email: tangyunlian@163.com.
}

\begin{abstract}
Background: Polo-like kinase 1 (PLK1) is a serine/threonine protein kinase, which has been studied as a potential gene therapy target for many years. PLK1 is overexpressed in a variety of tumors, and its expression often negatively correlated with patient prognosis. However, the role of PLK1 in nasopharyngeal carcinoma (NPC) is rarely studied.

Methods: Two recombinant vector plasmids were transfected into CNE2 cell lines by liposome transfection, CNE2/PLK1 shRNA target PLK1 mRNA, as well as a non-targeting control plasmid, CNE2/ NC shRNA. Meanwhile, non-transfected cells (CNE2) were also used as controls. Real-time quantitative PCR (qRT-PCR) and Western blotting were performed to detect the transfection effect. The effects of the downregulation of PLK1 on cell biological behavior was evaluated in vitro by using CCK8, Transwell, colony-forming and flow-cytometry assays.

Results: PLK1 mRNA and protein were significantly inhibited in CNE2/PLK1 shRNA cells. Compared to control groups, the CNE2/PLK1 shRNA cells showed slower cell growth and a significantly decreased cellcloning rate. Both migration and invasion were significantly inhibited in experimental cells. The proportions of G2-phase and apoptotic cells within the experimental group were significantly increased.

Conclusions: Our results indicate that specific interference of $P L K 1$ gene expression can significantly inhibit the proliferation and invasion of NPC (CNE2) cells.
\end{abstract}

Keywords: Polo-like kinase 1 (PLK1); nasopharyngeal carcinoma (NPC); down-regulation; targeted therapy; biological behavior

Submitted Feb 04, 2020. Accepted for publication Jul 14, 2020.

doi: $10.21037 /$ tcr-20-811

View this article at: http://dx.doi.org/10.21037/tcr-20-811

\section{Introduction}

Nasopharyngeal carcinoma (NPC) is a common malignant head and neck tumor, which is particularly prevalent in East and Southeast Asia. NPC is characterized by aggressive cellular behaviors such as early lymphatic spread and a strong predisposition to the formation of distant metastases (1). In recent years, with the improvement of the diagnosis and treatment of NPC, the incidence and mortality of NPC are gradually decreasing (2), but the prevalence of distant metastasis among patients with NPC is still as high as $14.1 \%$ (3). Thus, a great clinical need exists for the development of improved, individualized methods for the diagnosis and treatment of NPC.

Polo-like kinase 1 (PLK1) is a serine/threonine protein kinase, which has attracted widespread attention from 
Table 1 shRNA expression cassette

\begin{tabular}{ll}
\hline Name & Sequences \\
\hline PLK1 & S: 5'-CACCGGCAACCAAAGTCGAATATGATTCAAGAGATCATATTCGACTTTGGTTGCCTTTTTTG-3' \\
& A: 5'-GATCCAAAAAAGGCAACCAAAGTCGAATATGATCTCTTGAATCATATTCGACTTTGGTTGCC-3' \\
NC & S: 5'-CACCGTTCTCCGAACGTGTCACGTCAAGAGATTACGTGACACGTTCGGAGAATTTTTG-3' \\
& A: 5'-GATCCAAAAAATTCTCCGAACGTGTCACGTAATCTCTTGACGTGACACGTTCGGAGAAC-3' \\
\hline
\end{tabular}

researchers due to its important roles in the cell cycle. PLK1 is mainly involved in G2-M phase transition, promotes the formation of spindles, the maturation of centrosomes, the separation of sister chromosomes and the division of the cytoplasm (4). PLK1 is highly expressed in rapidly proliferating cells, such as tumor cells. Silencing the expression of PLK1 by antibodies, small molecular kinase inhibitors or RNAi (5) can effectively inhibit cell growth, induce G2-M phase arrest and cell apoptosis, but have no significant effect on normal cells, suggesting that PLK1 may be a candidate gene therapy target. Studies on acute leukemia have shown that PLK1 Thr210 hyperphosphorylation is closely related to poor prognosis in pediatric T-ALL, and PLK1-specific inhibitors can significantly inhibit the proliferation of leukemia cell lines, suggesting that targeted PLK1 inhibitors will provide new possibilities for the treatment of leukemia (5). Linton et al. found that RNAi-targeted PLK1 therapy showed promising results in malignant pleural mesothelioma (6). Furthermore, it was also shown that PLK1 is closely involved in the growth and proliferation of NPC cells (7-9).

In this study, the effects of down-regulating PLK1 expression on the biological behavior of CNE2 cells were examined in vitro several experiments. The role of PLK1 in the development of NPC was further elaborated to provide experimental evidence that could support novel approaches toward individualized diagnosis and targeted interventions in patients with NPC.

\section{Methods}

\section{Cell culture}

Human NPC cell line (CNE2) was donated by Cancer Research Institute, the Key Laboratory of Carcinogenesis and Cancer Invasion of the Chinese Ministry of Education, Central South University, Changsha, China. The cells were cultured in RPMI 1640 containing 10\% fetal bovine serum (FBS, Hangzhou Sijiqing Biological Engineering Materials
Co., Ltd., Hangzhou, China) and incubated in a constant temperature cell incubator at $37^{\circ} \mathrm{C}$ with $5 \% \mathrm{CO}_{2}$.

\section{Experimental methods}

\section{RNA interference and transfection}

Two recombinant vector plasmids, CNE2/PLK1 shRNA and CNE2/NC shRNA were designed and constructed by Shanghai Jema Bio Company (Shanghai, China). The shRNA expression cassette sequences for both constructs are shown in Table 1. The interfering- and the negative controlplasmid were transfected into CNE2 cells according to Lipofectamine $^{\mathrm{TM}} 2000$ liposome instructions (Invitrogen Inc., Carlsbad, CA, USA). After 48 hours, the cells were screened using the medium with a final concentration of $350 \mu \mathrm{g} / \mu \mathrm{L}$ G418 (AmrescoInc., Solon, USA), and cultured for 3-4 weeks until resistant clones with interference vectors appeared. Subsequently, the clones were expanded with a complete medium with a final concentration of $200 \mu \mathrm{g} / \mu \mathrm{L}$ G418.

\section{Western blotting analysis}

The total protein of the three groups were extracted using RIPA buffer (Beijing Kangwei Reagent Co., Ltd., Beijing, China). Then, proteins were quantified, isolated and transferred onto the PVDF membranes (Roche, Basel, Switzerland). The primary antibody of PLK1 (mouse-antihuman, Millipore Corp, Billerica, MA, USA) was diluted at $1: 1,000$, then uniformly dropped on the membrane, and left to incubate overnight at $4{ }^{\circ} \mathrm{C}$. After washing the membrane, a mouse secondary antibody (1:1,000, Sigma, USA) was incubated at room temperature for $1.5 \mathrm{~h}$ and then visualized using the Kangwei Colorimetric Kit (Beijing, China). At the same time, $\beta$-actin was established as an internal reference. Each sample was performed in triplicate.

\section{qRT-PCR}

Total RNA of each group was extracted, purified and reverse-transcribed into cDNA. The expression of PLK1 
Table 2 PCR primer sequences of gene

\begin{tabular}{ll}
\hline Gene & Sequence \\
\hline PLK1 & F: 5'-CAGCAAGTGGGTGGACTATT-3' \\
& R: 5'-GAGGATGAGGCGTGTTGAGT-3' \\
GAPDH & F: 5'-GGACCTGACCTGCCGTCTAG-3' \\
& R: 5'-TAGCCCAGGATGCCCTTGAG-3'
\end{tabular}

and internal standard GAPDH mRNA were amplified and detected by Applied Biosystems qRT-PCR instrument (USA). Briefly, heat activation $\left(95^{\circ} \mathrm{C}, 5 \mathrm{~min}\right)$, followed by 40 cycles of denaturation $\left(95^{\circ} \mathrm{C}, 10 \mathrm{~s}\right)$, extension $\left(60^{\circ} \mathrm{C}\right.$, $30 \mathrm{~s}$ ) and fluorescence collection at $72{ }^{\circ} \mathrm{C}$. Statistics of each well $\mathrm{Ct}$ value, computing $2^{-\Delta \Delta \mathrm{Ct}}$ value and analysis. The primers were designed and synthesized by Takara Biological Co. Ltd, China (Table 2). Each sample was performed in triplicate.

\section{Cell Counting Kit-8 assay}

Using 96-well plates (Costar, CA, USA), 1,000 cells per well (three replicate wells per group) were inoculated into the plates and cultured for a period of between one and 6 days. Add $100 \mu \mathrm{L}$ mixture (CCK-8 solution $10 \mu \mathrm{L}+1640$ medium $90 \mu \mathrm{L}$ ) to each well and incubate for one hour. The $450-\mathrm{nm}$ absorbance values were measured by a microplate reader (Multiskan MK3, Finland Labsystem).

\section{Colony formation assay}

Using six-well plates, 300 cells per well (three replicate wells per group) were inoculated into the plates and cultured for two weeks. Two weeks later, macroscopic clones were formed and cell culture was terminated. Clones with more than 50 cells were fixed, stained, and counted.

\section{Migration and invasion assays}

Matrigel ${ }^{\circledR}$ Matrix (Corning Costar, Manassas, VA, USA) and Transwell chamber with $8-\mu \mathrm{m}$ aperture (Corning Costar) were used for Transwell assays. In the migration experiment, cells of the experimental and other two control groups were starved for 6 hours, detached with $0.25 \%$ trypsin, and suspended in serum-free medium at a concentration of $2 \times 10^{5}$ cells $/ \mathrm{mL}$. After $700 \mu \mathrm{L}$ of the basal medium was added to the lower chamber, and cell suspension $(150 \mu \mathrm{L})$ was added to the upper layer of Transwell chamber. After incubation for 48 hours, the chamber was removed. Then, the cells passing through the filtration membrane were fixed, stained and counted under a microscope.

For the invasion experiment, a Matrigel layer was applied to the bottom of the upper chamber first. Briefly, serumfree medium with Matrigel at $6: 1$ was mixed, $50 \mu \mathrm{L}$ of the diluted Matrigel ${ }^{\circledR}$ Matrix was taken to the center of the upper chambers, and was incubated overnight at $37^{\circ} \mathrm{C}$. The subsequent steps were identical to those of the migration experiment.

\section{Flow cytometry}

The cell density of each group was adjusted to $2 \times 10^{6}$ cells $/ \mathrm{mL}$. Then, cells were fixed overnight with $70 \%$ cold ethanol at $4{ }^{\circ} \mathrm{C}$. The cell cycle was monitored by Well Biological Science (Changsha, China). It was measured using a flow cytometer (Beckman, Coulter) and analyzed by ModFit software (Beckman, Coulter).

\section{Statistical analysis}

GraphPad Prism 5 software was used for data analysis, all data were expressed as mean \pm SD. All experimental data were analyzed by non-parametric test. Differences were considered statistically significant for $\mathrm{P}$ value $<0.05$.

\section{Results}

\section{Successful construction of a stable and down-regulated PLK1 in CNE2 cell line}

Fluorescence microscopy showed that the experimental group (transfected with CNE2/PLK1 shRNA) and negative control group (transfected with CNE2/NC shRNA) appeared strong green fluorescence signals and that the transfection efficiency was over 90\% (Figure 1), compared with CNE2 group (the blank group). The results from Western blotting analysis and qRT-PCR showed that PLK1 was significantly down-regulated in protein (Figure $2 A, B$ ) and mRNA (Figure 2C) levels in CNE2/PLK1 shRNA cells compared with CNE2/NC shRNA or CNE2 groups. These results suggested that a CNE2/PLK1 shRNA cell line with down-regulation of PLK1 was successfully constructed.

\section{Effects of down-regulation PLK1 expression on biological behaviors of CNE2 cells}

Silencing PLK1 inhibited cell growth and proliferation in CNE2 cells

The results in the plate cloning experiment showed that the 


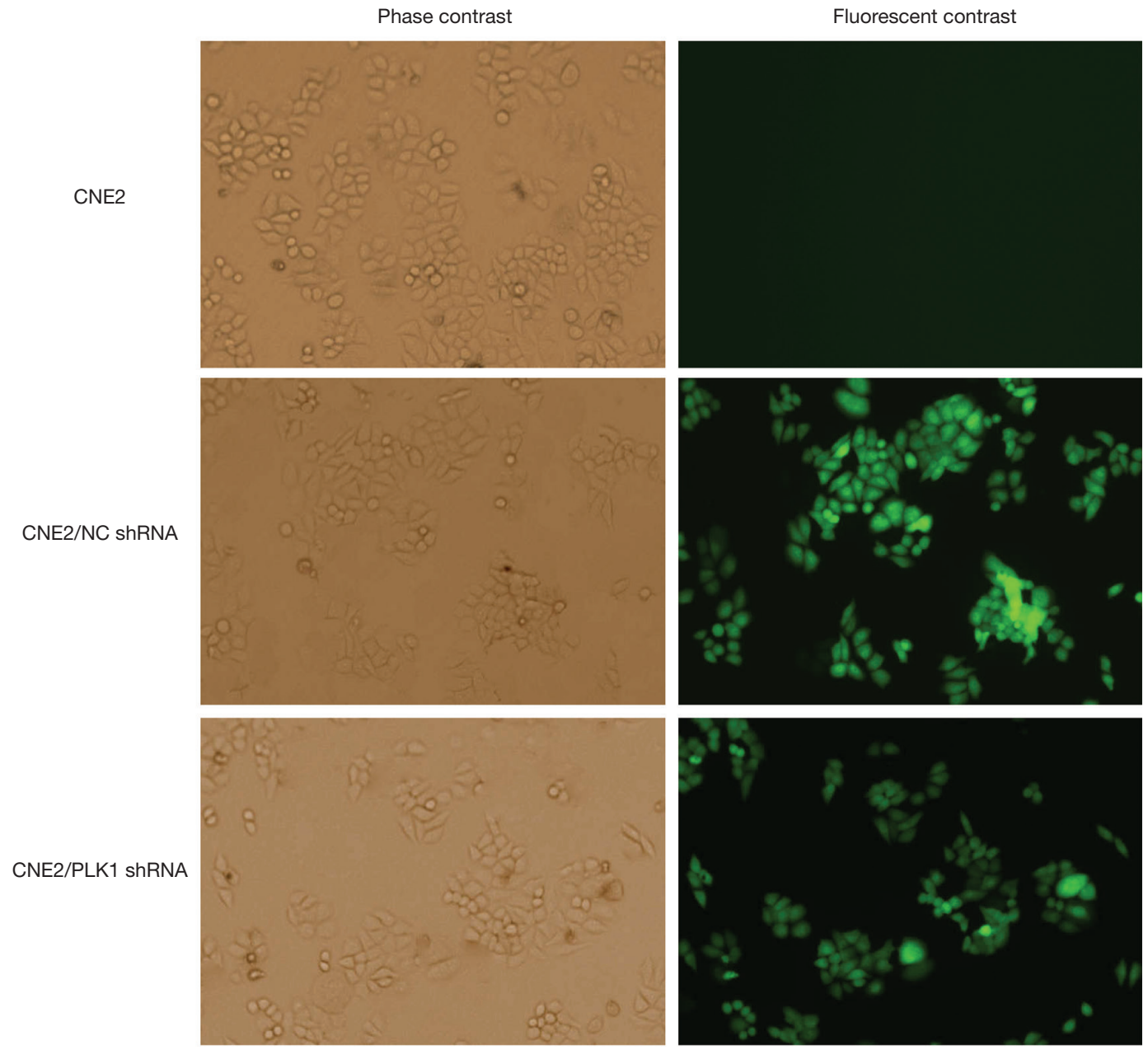

Figure 1 Fluorescence expression in each group (100× magnification). Both CNE2/PLK1 shRNA group and CNE2/NC shRNA group appeared strong green fluorescence signals, and the transfection efficiency was over $90 \%$, while no fluorescence signal appeared in the CNE2 group. PLK1, Polo-like kinase 1.

cloning formation rate of CNE2/PLK1 shRNA group was $23.67 \%$, while the rates of CNE2/NC shRNA and CNE2 groups were $57.9 \%$ and $62.76 \%$, respectively (Figure $3 A, B$ and Table 3). CCK-8 assay results showed the growth rate of CNE2 cells transfected with PLK1-interfering plasmid was significantly slower than CNE2/NC shRNA or CNE2 groups (Figure $3 C$ ) from the third to the fifth day. These results suggested that silencing PLK1 could inhibit growth and proliferation in the CNE2 cell.

\section{Silencing PLK1 inhibited migration and invasion of CNE2 cells}

The results of Transwell assay showed that the number of migrating (Figure $4 A$ ) and invading (Figure $4 B$ ) cells in CNE2/PLK1 shRNA group were 32.00 \pm 2.07 (cells,

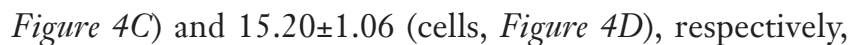
which was much lower than that in CNE2/NC shRNA or CNE2 groups, indicating that silencing PLK1 could inhibit migration and invasion in the CNE2 cell.

\section{Silencing PLK1 induced G2 cell-cycle arrest in CNE2 cells}

Flow cytometry results showed that compared with CNE2/NC shRNA or CNE2 groups, the proportion of G2 phase and apoptotic cells in CNE2/PLK1 shRNA group were increased, while the proportion of $S$ phase cells was significantly decreased (Figure 5 and Table 4). This suggested that down-regulation of PLK1 expression could induce G2 phase arrest and apoptosis in CNE2 cells. 

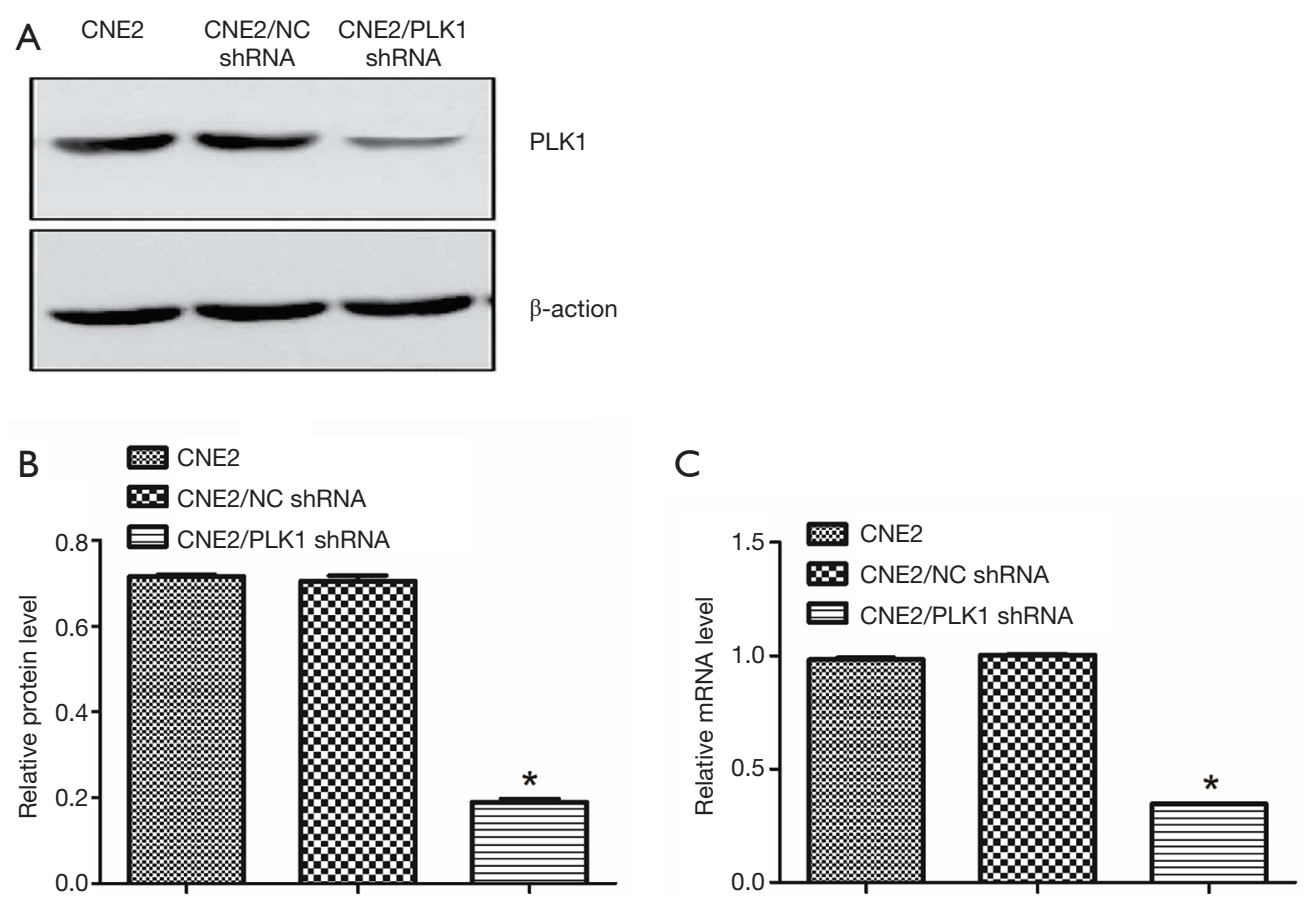

Figure 2 Protein and mRNA expression of PLK1 in CNE2 cells. (A) PLK1 protein expression levels in CNE2 cells were examined by Western blotting; (B) ratio of gray value of PLK1 protein expression levels in CNE2 cells; (C) histogram of PLK1 mRNA expression levels in CNE2 cells. Compared with the control groups, PLK1 protein and mRNA expression in CNE2/PLK1 shRNA group was significantly down-regulated. *, $\mathrm{P}<0.05$. PLK1, Polo-like kinase 1.

\section{Discussion}

The Polo-like kinase (PLKs) family is a highly conserved serine/threonine kinase family, which is involved in the regulation of cell proliferation and survival (10). In mammals, PLKs family includes at least five members (PLK1-5). All PLKs are highly homology in molecular structure: a kinase domain (KD) and two or more Polobox domains (PBD) (11). PLK1's PBD regulates PLK1's position. The $\mathrm{KD}$ is a serine/threonine $\mathrm{KD}$ with a T-loop. Phosphorylation of Thr210 in the T-loop is closely related to the activation of PLK1. PLK1 has varying levels of activity at different stages of the cell cycle. It is barely expressed during the G0/G1 phase, which begins to accumulate during the $S$ phase, reaches a peak during the $M$ phase, after which it is drastically reduced after cell division. This pattern reflects the important role that PLK1 plays in mitosis, making it one of the markers of cells' ability to proliferate. In addition, the subcellular location of PLK1 changes with cell cycle progression. In the early stage of mitosis, PLK1 is mainly concentrated in the centrosomes and gradually migrates from the spindle to the equatorial plate during mitosis. In the late stage, PLK1 accumulates in the mid-body to assist cytoplasmic division (12).

Mitotic entry depends on the activation of Cdk1-cyclin B complex. The activity of Cdk1-cyclin B complex is regulated by the upstream phosphatase activators, Cdc25A, Cdc25B, Cdc25C and the inhibition kinases, Wee1 and Myt1 (13). PLK1 is the key regulatory factor for the initiation of mitosis. Specifically, PLK1-phosphorylated Cdc25 translocates to the nucleus, resulting in the release of two inhibition phosphorylation sites (thr14 and thr15) on the Cdk1-ATP binding domain. Moreover, PLK1 inhibits Wee1 and Myt1 activities and promotes the activation of Cdk1cyclin B (14). Further evidence indicates that PLK1 directly phosphorylates cyclin $\mathrm{B}$, although it remains to be clarified whether this leads to the nuclear positioning of cyclin $\mathrm{B}$ and the activation of the Cdk1-cyclin B complex (15). However, these studies have supported a key role of PLK1 in the G2/ $\mathrm{M}$ transformation. In vitro studies of gastric tumors showed that inhibiting the expression of PLK1 caused a severe G2/ $M$ cell-cycle arrest in tumor cells (16). Our results confirm that CNE2 cells show significant G2 cell-cycle arrest and decelerated cell growth and proliferation in response to 
A
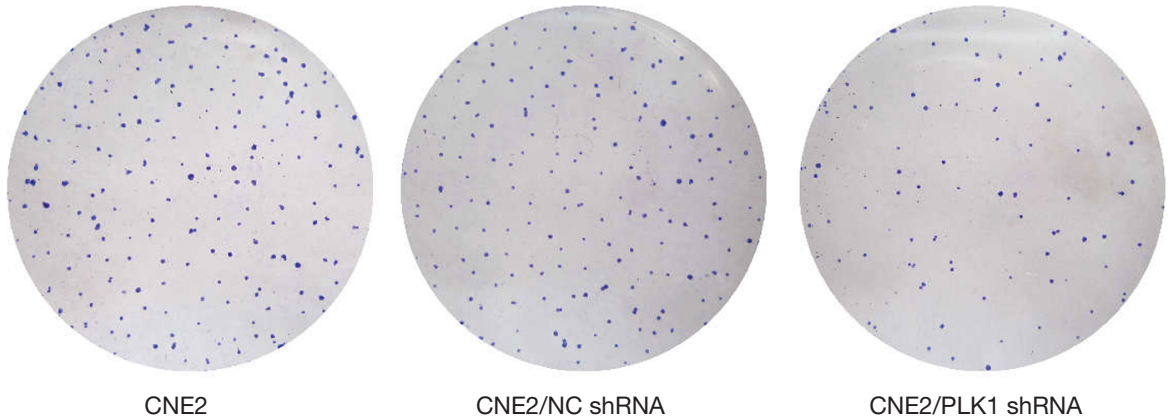

CNE2/NC shRNA

CNE2/PLK1 shRNA
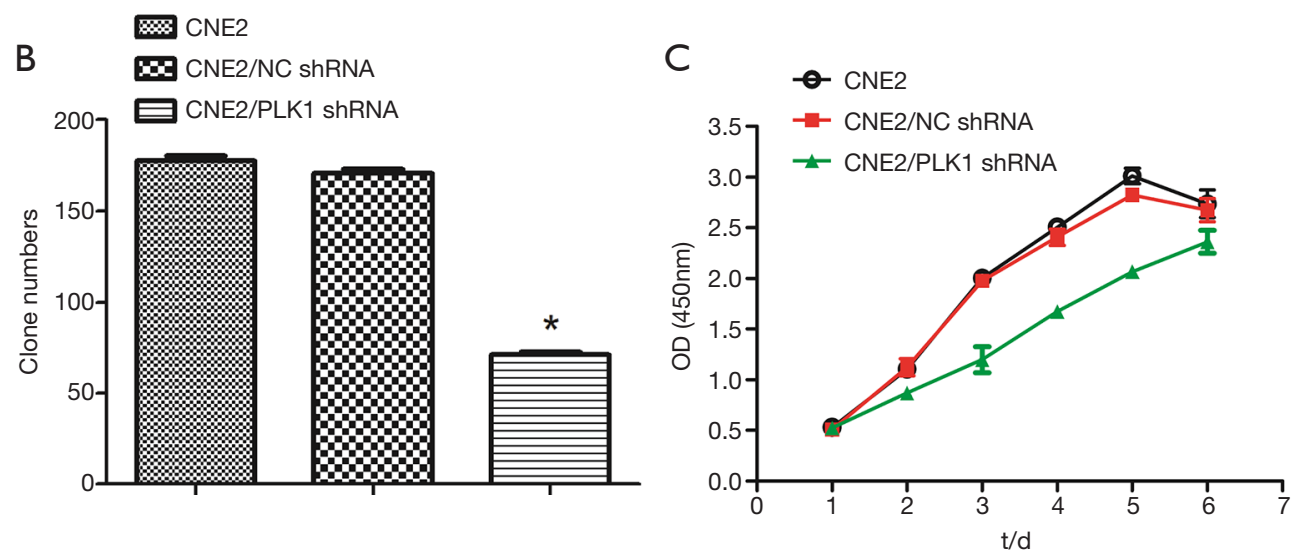

Figure 3 Silencing PLK1 inhibited cell growth and proliferation in CNE2 cells. (A) Colony formation of three groups (Giemsa staining); (B) histogram of clone numbers in the three groups, compared to control groups, the CNE2/PLK1 shRNA cells showed a significantly decreased cell-cloning rate; (C) CCK-8 assay was conducted and absorbance of CNE2 cells at $450 \mathrm{~nm}$ was compared, indicating the number of active cells in the CNE2, CNE2/PLK1 shRNA and CNE2/NC shRNA groups. The cell-growth rate of the CNE2/PLK1 shRNA group was significantly slower than those in the CNE2/NC shRNA group or CNE2 group from the third to the fifth day. *, P<0.05. PLK1, Polo-like kinase 1 .

Table 3 Colony formation of three groups

\begin{tabular}{lccc}
\hline Variable & CNE2 & $\begin{array}{c}\text { CNE2/NC } \\
\text { shRNA }\end{array}$ & $\begin{array}{c}\text { CNE2/PLK1 } \\
\text { shRNA }\end{array}$ \\
\hline $\begin{array}{l}\text { Number of } \\
\text { inoculated cells }\end{array}$ & 300 & 300 & 300 \\
$\begin{array}{l}\text { Number of clones } \\
\text { Formation rates of } \\
\text { clones }\end{array}$ & $62.76 \%$ & $57.9 \%$ & $23.67 \%{ }^{*}$ \\
\hline
\end{tabular}

The difference was statistically significant compared with the control groups CNE2 /NC shRNA or CNE2. * $\mathrm{P}<0.05$.

down-regulation of PLK1 expression. Wachowicz et al. (17) showed that knocking down PLK1 expression in pregnant mice was associated with embryonic lethality. Furthermore, inhibition of PLK1 expression induced abnormal mitosis and even cell death.

PLK1 is overexpressed in a variety of tumors, and its expression often negatively correlated with patient prognosis (18). However, the mechanism in which PLK1 overexpression induces tumorigenesis has so far not been identified. It may be related to several factors. Firstly, PLK1 is directly involved in the malignant transformation of cells. Exogenous PLK1 transfected into normal fibroblasts can lead to cell malignancy and tumor formation in nude mice (19). Secondly, PLK1 can inhibit or down-regulate the expression of tumor suppressor genes. Most notably, suppressor gene p53 is considered to be the "guardian of the genome" and mainly involved in inhibiting abnormal cell proliferation. Ando et al. found that PLK1 can bind directly to $\mathrm{p} 53$, inhibiting $\mathrm{p} 53$-mediated transcription and pro-apoptotic function (20). In addition, PLK1 can phosphorylate G2 and S-phase-expressed-1 (GTSE1) at Ser435. This protein is able to downregulate p53 following translocation to the nucleus. After GTSE1 binds to p53 and shuttles it out of the nucleus, p53 is degraded (21). PLK1 also interacts with tumor suppressor genes such as CHK2, BRCA 1/2, ATM, BUBR1, REST, and TSC1/2D 


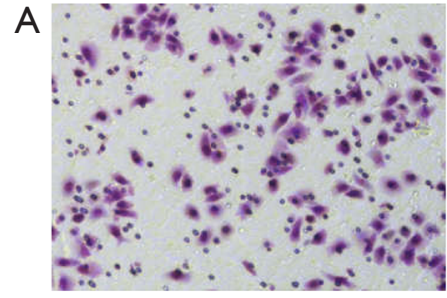

CNE2

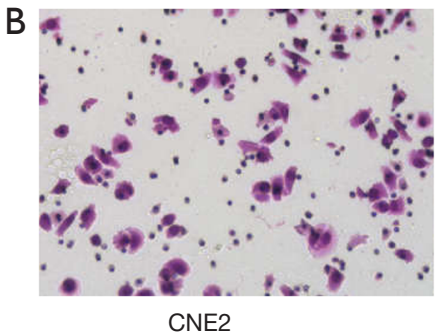

C

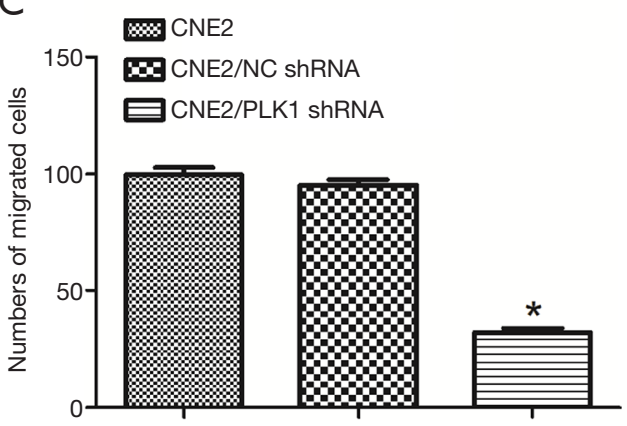

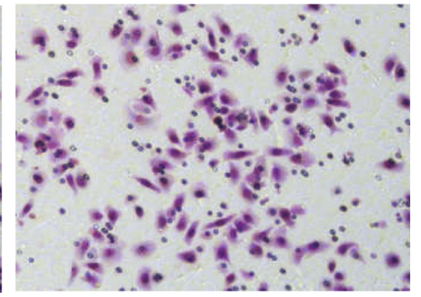

CNE2/NC ShRNA

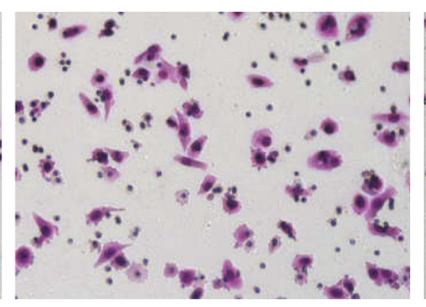

CNE2/NC ShRNA

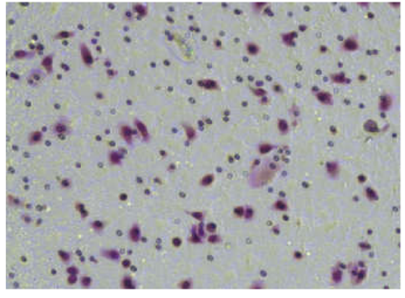

CNE2/PLK1 ShRNA

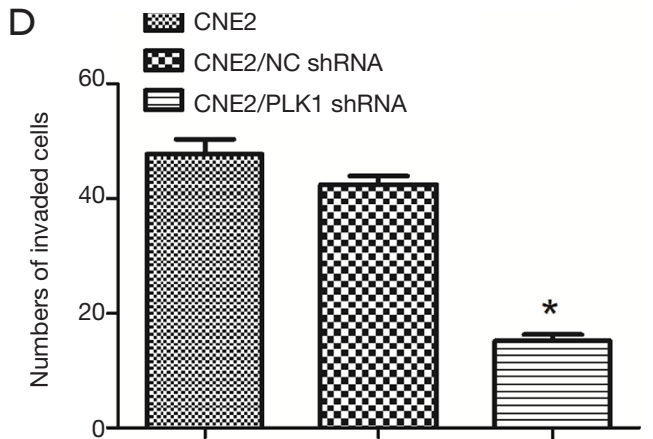

Figure 4 Silencing PLK1 inhibited CNE2-cells migration and -invasion. (A) Migration of cells in the three groups (100× magnification, Giemsa staining); (B) invasion of cells in the three groups (100x magnification, Giemsa staining); (C) histogram of migration cell numbers in the three groups; and (D) histogram of invasion cells within the three groups. Compared with the control groups, the migration and invasion ability of CNE2/PLK1 shRNA cells were significantly reduced. * $\mathrm{P}<0.05$. PLK1, Polo-like kinase 1.

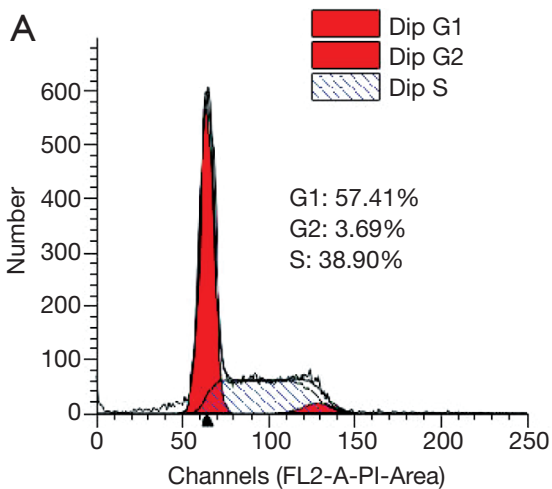

CNE2

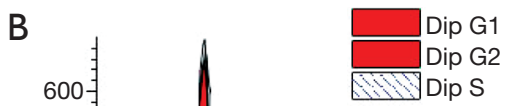

G1: $58.52 \%$

G2: $3.25 \%$

S: $38.24 \%$

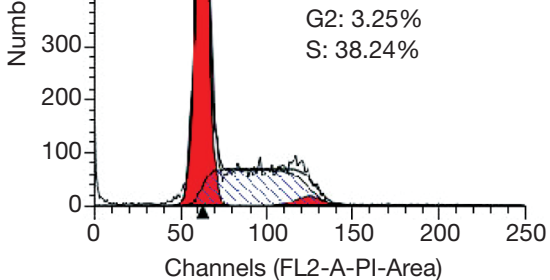

CNE2/NC ShRNA

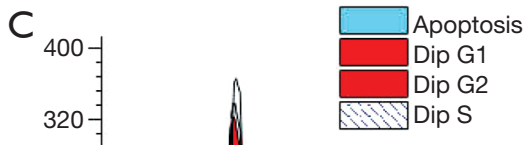

G1: $61.56 \%$

G2: $10.65 \%$

S: $27.79 \%$

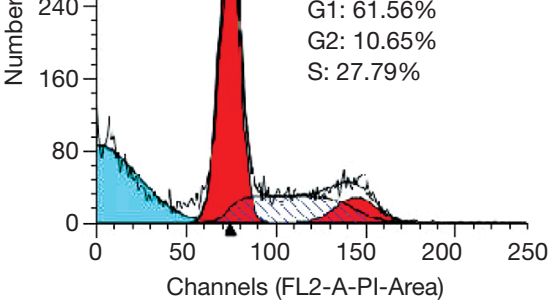

CNE2/PLK1 ShRNA

Figure 5 Cell-cycle distribution as analyzed by flow cytometry. The proportion of G2 phase and apoptotic cells in the CNE2/PLK1 shRNA group were significantly increased, while the proportion of $\mathrm{S}$ phase cells was significantly decreased, compared with the CNE2/NC shRNA or CNE2 groups. PLK1, Polo-like kinase 1. 
Table 4 Distribution of cell cycle

\begin{tabular}{lccc}
\hline \multirow{2}{*}{ Groups } & \multicolumn{3}{c}{ Distribution of cell cycle (\%) } \\
\cline { 2 - 4 } & $\mathrm{G} 1$ & $\mathrm{G} 2$ & $\mathrm{~S}$ \\
\hline CNE2 & $57.59 \pm 0.12$ & $3.50 \pm 0.13$ & $38.68 \pm 0.22$ \\
CNE2/NC shRNA & $59.22 \pm 0.47$ & $3.53 \pm 0.16$ & $38.82 \pm 0.23$ \\
CNE2/PLK1 shRNA & $60.40 \pm 0.36$ & $10.94 \pm 0.16^{\star}$ & $27.81 \pm 0.11^{\star}$ \\
\hline
\end{tabular}

Statistically significant difference compared with the control groups CNE2/NC shRNA or CNE2. * $\mathrm{P}<0.05$.

by promoting DNA replication and division of damaged cells, thereby stimulating tumorigenesis (22). Finally, PLK1 can affect signal pathways related to tumor formation. It is estimated that PLK1 participates in $75 \%$ of the signaling pathways mediating tumorigenesis and development (23). Recent studies have found that PLK1 can phosphorylate and stabilize Krüppel-like factor 4 (KLF4), promoting KLF4-mediated carcinogenesis in NPC (8).

Early lymphatic spread and a strong disposition to the formation of distant metastasis are characteristic behaviors of NPC cells (24). The epithelium-to-mesenchymal transformation (EMT) contributes to tumors' metastatic activities. By means of complex molecular changes, epithelial tumor cells are transformed into more migratory interstitial cells. These properties of tumor cells facilitate metastasis. Following the transfection of a lentiviral vector containing PLK1 cDNA into normal prostate epithelial cells (RWPE-1), the overexpression of PLK1 led to the transformation from epithelioid to fusiform cells in an experimental group of mice injected with RWPE-1-PLK1 cells (25). Moreover, the extent of cell migration was significantly enhanced, suggesting that EMT did occur in the cells. Subsequent experiments on neoplasms induced in nude mice showed that neoplasms were successfully induced in the experimental group that was injected with RWP-1-PLK1. It was interesting that five of seven mice developed micrometastasis with tumors in lungs. It was further confirmed that PLK1 can promote the invasion and metastasis of tumor cells by inducing cell EMT (26). In another study, down-regulation of the expression of PLK1 significantly enhanced inhibition of proliferation, independent growth, invasion and metastasis of tumor cells by RNAi and small-molecule inhibitors in colorectal, breast and thyroid cancers (27). In our study, an RNA interference technique was used. The results showed that the ability to migrate and invade was also significantly reduced in CNE2 cells after the silencing of PLK1 gene.
With the growing recognition of PLK1's functions, an increasing number of researchers have begun to pay attention to the potential of PLK1 as a target for tumor gene therapy. Thus far, a number of small-molecule inhibitors of PLK1 have been developed, of which some have entered clinical trial stage III and achieved breakthrough progress (28). Compared with traditional chemotherapy and radiotherapy, PLK1 inhibitors show considerable antitumor specificity. In addition, PLK1 inhibitors combined with traditional chemotherapy or radiotherapy may enhance the sensitivity of tumor cells to chemotherapy or radiotherapy. This synergy was observed when the PLK1 selective inhibitor, BI2536, was co-administered with cisplatin to gastric cancer cells (29). Li et al. found that the synergistic effect of BI2536 and Aurora kinase inhibitor can significantly enhance mitotic catastrophe in NPC cells (9). PLK1 inhibitors were also found to enhance the sensitivity to radiotherapy of non-small-cell lung cancer (30). Radiotherapy is still the main treatment for nasopharyngeal cancer, but unfortunately, the failure rate ranges from $7 \%$ to $58 \%$ (31). Thus, PLK1 inhibitors combined with radiotherapy may have the potential to increase the sensitivity to radiotherapy for nasopharyngeal cancer.

\section{Conclusions}

Our results demonstrate that specific interference of PLK1 gene expression can significantly inhibit the proliferation and invasion of NPC cells. This may provide an experimental basis for the application of PLK1 target inhibitors in the treatment of NPC.

\section{Acknowledgments}

We thank Li Cao (Cancer Research Institute, the Key Laboratory of Carcinogenesis and Cancer Invasion of the Chinese Ministry of Education, School of Basic Medical Science, Central South University, Changsha, China) for kindly providing CNE2 cell line.

Funding: This study was supported by the National Natural Science Foundation of China (No. 81272182), the Key Project of Hunan Provincial Education Department (16K077, 17K081), Hunan Province Key Laboratory of Tumor Cellular \& Molecular Pathology (2016TP1015).

\section{Footnote}

Data Sharing Statement: Available at http://dx.doi. 
org/10.21037/tcr-20-811

Conflicts of Interest: All authors have completed the ICMJE uniform disclosure form (available at http://dx.doi. org/10.21037/tcr-20-811). All authors report grants from the National Natural Science Foundation of China (No.81272182), grants from the Key Project of Hunan Provincial Education Department (16K077, 17K081), grants from Hunan Province Key Laboratory of Tumor Cellular \& Molecular Pathology (2016TP1015), during the conduct of the study.

Ethical Statement: The authors are accountable for all aspects of the work in ensuring that questions related to the accuracy or integrity of any part of the work are appropriately investigated and resolved.

Open Access Statement: This is an Open Access article distributed in accordance with the Creative Commons Attribution-NonCommercial-NoDerivs 4.0 International License (CC BY-NC-ND 4.0), which permits the noncommercial replication and distribution of the article with the strict proviso that no changes or edits are made and the original work is properly cited (including links to both the formal publication through the relevant DOI and the license). See: https://creativecommons.org/licenses/by-nc-nd/4.0/.

\section{References}

1. Tang LQ, Lu TY, Li Y, et al. Patterns of Failure and Survival Trends Of 720 Patients with Stage I Nasopharyngeal Carcinoma Diagnosed from 1990-2012: A Large-scale Retrospective Cohort Study. J Cancer 2018;9:1308-17.

2. Chen YP, Chan ATC, Le QT, et al. Nasopharyngeal carcinoma. Lancet 2019;394:64-80.

3. Yang Y, Wang X, Yang J, et al. Loss of ARID1A promotes proliferation, migration and invasion via the Akt signaling pathway in NPC. Cancer Manag Res 2019;11:4931-46.

4. Colicino EG, Hehnly H. Regulating a key mitotic regulator, polo-like kinase 1 (PLK1). Cytoskeleton (Hoboken) 2018;75:481-94.

5. Goroshchuk O, Kolosenko I, Vidarsdottir L, et al. Pololike kinases and acute leukemia. Oncogene 2019;38:1-16.

6. Linton A, Cheng YY, Griggs K, et al. An RNAi-based screen reveals PLK1, CDK1 and NDC80 as potential therapeutic targets in malignant pleural mesothelioma. $\mathrm{Br}$ J Cancer 2018;118:e13.
7. Cheung AK, Ip JC, Lung HL, et al. Polo-like kinase inhibitor Ro5203280 has potent antitumor activity in nasopharyngeal carcinoma. Mol Cancer Ther 2013;12:1393-401.

8. Mai J, Zhong ZY, Guo GF, et al. Polo-Like Kinase 1 phosphorylates and stabilizes KLF4 to promote tumorigenesis in nasopharyngeal carcinoma. Theranostics 2019;9:3541-54.

9. Li J, Hong MJ, Chow JP, et al. Co-inhibition of polo-like kinase 1 and Aurora kinases promotes mitotic catastrophe. Oncotarget 2015;6:9327-40.

10. Lin SF, Lin JD, Yeh CN, et al. Targeting PLKs as a therapeutic approach to well-differentiated thyroid cancer. Endocr Relat Cancer 2019;26:727-38.

11. Zitouni S, Nabais C, Jana SC, et al. Polo-like kinases: structural variations lead to multiple functions. Nat Rev Mol Cell Biol 2014;15:433-52.

12. Petronczki M, Lénárt P, Peters JM. Polo on the Risefrom Mitotic Entry to Cytokinesis with Plk1. Dev Cell 2008;14:646-59.

13. Gheghiani L, Loew D, Lombard B, et al. PLK1 Activation in Late G2 Sets Up Commitment to Mitosis. Cell Rep 2017;19:2060-73.

14. Lu LY, Wood JL, Minter-Dykhouse K, et al. Polo-like kinase 1 is essential for early embryonic development and tumor suppression. Mol Cell Biol 2008;28:6870-6.

15. Linder MI, Köhler M, Boersema P, et al. Mitotic Disassembly of Nuclear Pore Complexes Involves CDK1- and PLK1Mediated Phosphorylation of Key Interconnecting Nucleoporins. Dev Cell 2017;43:141-56.e7.

16. Dang SC, Fan YY, Cui L, et al. PLK1 as a potential prognostic marker of gastric cancer through MEKERK pathway on PDTX models. Onco Targets Ther 2018;11:6239-47.

17. Wachowicz P, Fernández-Miranda G, Marugán C, et al. Genetic depletion of Polo-like kinase 1 leads to embryonic lethality due to mitotic aberrancies. Bioessays 2016;38 Suppl 1:S96-S106.

18. Yang X, Mo W, Fang Y, et al. Up-regulation of Pololike Kinase 1 in nasopharyngeal carcinoma tissues: a comprehensive investigation based on RNA-sequencing, gene chips, and in-house tissue arrays. Am J Transl Res 2018;10:3924-40.

19. Smith MR, Wilson ML, Hamanaka R, et al. Malignant transformation of mammalian cells initiated by constitutive expression of the polo-like kinase. Biochem Biophys Res Commun 1997;234:397-405.

20. Ando K, Ozaki T, Yamamoto H, et al. Polo-like kinase 1 
(Plk1) inhibits $\mathrm{p} 53$ function by physical interaction and phosphorylation. J Biol Chem 2004;279:25549-61.

21. Liu XS, Li H, Song B, et al. Polo-like kinase 1 phosphorylation of G2 and S-phase-expressed 1 protein is essential for p53 inactivation during G2 checkpoint recovery. EMBO Rep 2010;11:626-32.

22. Wang L, Guo Q, Fisher LA, et al. Regulation of pololike kinase 1 by DNA damage and PP2A/B55 $\alpha$. Cell Cycle 2015;14:157-66.

23. Cholewa BD, Liu X, Ahmad N. The role of polo-like kinase 1 in carcinogenesis: cause or consequence. Cancer Res 2013;73:6848-55.

24. Sun XS, Liu DH, Liu SL, et al. Patterns of Failure and Survival Trends in 3,808 Patients with Stage II Nasopharyngeal Carcinoma Diagnosed from 1990 to 2012: A Large-Scale Retrospective Cohort Study. Cancer Res Treat 2019;51:1449-63.

25. Wu J, Ivanov AI, Fisher PB, et al. Polo-like kinase 1 induces epithelial-to-mesenchymal transition and promotes epithelial cell motility by activating CRAF/ERK signaling. Elife 2016;5:e10734.

26. Fu Z, Wen D. The Emerging Role of Polo-Like Kinase

Cite this article as: Zhou Y, Wu C, Liu B, Zhu J, Zhong Y, Yuan Y, Huang Y, Tang Y. shRNA targeting PLK1 inhibits the proliferation and invasion of nasopharyngeal carcinoma cells. Transl Cancer Res 2020;9(9):5350-5359. doi: 10.21037/tcr-20-811
1 in Epithelial-Mesenchymal Transition and Tumor Metastasis. Cancers (Basel) 2017;9:131.

27. Han DP, Zhu QL, Cui JT, et al. Polo-like kinase 1 is overexpressed in colorectal cancer and participates in the migration and invasion of colorectal cancer cells. Med Sci Monit 2012;18:BR237-46.

28. Van den Bossche J, Lardon F, Deschoolmeester V, et al. Spotlight on Volasertib: Preclinical and Clinical Evaluation of a Promising Plk1 Inhibitor. Med Res Rev 2016;36:749-86.

29. Lian G, Li L, Shi Y, et al. BI2536, a potent and selective inhibitor of polo-like kinase 1 , in combination with cisplatin exerts synergistic effects on gastric cancer cells. Int J Oncol 2018;52:804-14.

30. Yao D, Gu P, Wang Y, et al. Inhibiting polo-like kinase 1 enhances radiosensitization via modulating DNA repair proteins in non-small-cell lung cancer. Biochem Cell Biol 2018;96:317-25.

31. Yan H, Cao X, Wang J. Application of intensity-modulated radiation therapy in the treatment of nasopharyngeal carcinoma. Oncol Lett 2017;14:7773-6. 